\title{
Early Fetal Death
}

National Cancer Institute

\section{Source}

National Cancer Institute. Early Fetal Death. NCI Thesaurus. Code C112862.

Death of a fetus at 10 weeks, 0 days to 15 weeks, 6 days of gestation. 